\title{
DESCENTRALIZACIÓN DEL ESTADO Y TERREMOTO: EL CASO DE CHILE
}

\author{
LEONARdo Letelier S. (lletelie@iap.uchile.cl) \\ Instituto de Asuntos Públicos - Universidad de Chile
}

El presente artículo ofrece un marco analítico dentro del cual racionalizar y optimizar la gestión de catástrofes. El modelo planteado se basa en argumentos económicos tradicionales que justifican la acción del Estado, los cuales son puestos en el contexto de una función de producción de "seguridad ante catástrofes". Se examina el caso del terremoto de Chile del 27 de febrero del 2010, haciendo especial énfasis en la "tecnología" implícita en el manejo de sus efectos y las debilidades en la etapa de prevención.

Palabras clave: Gestión de desastres; terremoto; Chile.

\section{DECENTRALIZATION AND EARTHQUAKE: THE CASE OF CHILE}

This paper provides an analytical framework in which to analyze and optimize disaster management. The model being proposed is based on traditional economic arguments that justify State action in the context of a production function which delivers "security in face of catastrophes". It examines the case of the Chilean earthquake of 27 February, 2010, emphasizing the implicit role of "technology" in managing potential effects and weaknesses at the prevention stage.

Keywords: disaster management; earthquake; Chile. 


\section{INTRODUCCIÓN}

Este trabajo ofrece un marco analítico general dentro del cual racionalizar y optimizar la gestión de catástrofes. El modelo planteado se basa en los argumentos económicos tradicionales que justifican la acción del Estado, los cuales son puestos en el contexto de una función de producción de "seguridad ante catástrofes". En este marco se examina el caso del terremoto de Chile del 27 de febrero del 2010, haciendo especial énfasis en la "tecnología" implícita en el manejo de sus efectos y las debilidades en la etapa de prevención. Dicha tecnología debe entenderse como la conjunción de la propia acción del Estado más la correspondiente colaboración voluntaria de la sociedad civil. El análisis realizado entiende que la acción del Estado tiene una dimensión central en la asignación de funciones y responsabilidades entre niveles de gobierno. Ello supone reconocer que si bien la descentralización de funciones en el tema que nos ocupa permite potenciar la generación de información a favor de una intervención más oportuna y focalizada, esta también conlleva el peligro de la descoordinación entre unidades de gestión independientes dentro del territorio nacional.

El resto del trabajo está organizado como sigue. La sección 1 aborda las consideraciones teóricas del tema, plantea un modelo general y discute sobre la asignación óptima de funciones entre niveles de gobierno. La sección 2 presenta algunos casos emblemáticos en la experiencia internacional. El caso específico de un terremoto y la experiencia de Chile a la luz del episodio del 27 de febrero son abordados en la sección 3. La última sección presenta las conclusiones.

\section{EL APORTE DE LA TEORÍA}

\subsection{La administración de una catástrofe desde la perspectiva económica}

\section{Contexto General}

La "administración de una catástrofe", vista desde la perspectiva de la acción del Estado, supone reconocer insumos, costos y resultados en el proceso de prevenir -por una parte- y socorrer - por la otra- los efectos de la misma. El ángulo analíti- 
co del problema exige identificar y comprender el proceso productivo subyacente, el cual se aparta en varios y fundamentales aspectos respecto de otros ámbitos de la acción del Estado. Debe existir por lo tanto una función de producción, cuyo "output" se expresa en el grado de impacto que la acción referida logra en términos físicos y humanos. Una gestión exitosa será aquella que minimice el efecto negativo de la catástrofe, sujeto a las restricciones propias de cada caso.

Bajo el supuesto de que toda comunidad está expuesta a una situación de catástrofe, esta debe resolver dos problemas centrales que son inherentes a toda política pública. El primero es la magnitud de los recursos destinados a la prevención y gestión de la misma. La respuesta a tan importante pregunta estará severamente condicionada por el costo de oportunidad de estos recursos, y por lo tanto por el grado de desarrollo del país en cuestión. No es extraño, ni tampoco "irracional", que las consecuencias del terremoto en Haití hayan sido tanto más devastadoras que las de Chile como fruto del terremoto del 27 de febrero, habiendo sido el primero un evento de menor magnitud desde el punto de vista geológico. El beneficio de la prevención, bajo la forma de mayores estándares en la construcción y más recursos comprometidos en la atención de las víctimas, supone ingentes sacrificios para un país - como Haití- en que una parte significativa de la población no alcanza un nivel de acceso mínimamente aceptable en su estándar de vida.

Habiéndose establecido el monto socialmente óptimo de los recursos, la segunda cuestión consiste en determinar la forma en la cual se van a utilizar, y más específicamente, cómo se debe articular la gestión pública tanto en la etapa de la prevención como en aquella de la atención de los afectados. El foco de este ensayo se centra sobre este segundo problema, bajo el entendido de que un rasgo central del resultado, depende críticamente de la asignación de competencias y responsabilidades al interior del aparato público. En la esfera de la prevención, se requiere una interacción permanente e informada entre el (los) organismo(s) público(s) competente(s), y un diseño eficiente y efectivo en el esfuerzo de anticipar los eventuales efectos de una catastrofe. Dicho propósito exige un difícil ejercicio de coordinación tanto al interior del Estado como así también con diversas organizaciones propias de la sociedad civil, una clara delimitación de las acciones urgentes respecto de aquellas más vinculadas a la reconstrucción, y una estructura organizativa que sirva eficientemente las exigencias de información, abastecimiento y logística que se requieren. 


\section{Marco Analítico}

A fin de abordar sistemáticamente el tema, debemos establecer ciertos principios generales que son transversales en cualquier política pública. El primero es que todos los objetivos explícitos -e implícitos- de la acción del Estado, contienen múltiples dimensiones específicas que deben ser atendidas en forma coherente y articulada. Esta multidimensionalidad está presente en la provisión de todo bien o servicio púbico, cualesquiera sea su naturaleza. De la correcta identificación de tales dimensiones, y del apropiado diseño de intervención depende el éxito de la política. Corresponde pues identificar cuáles son las dimensiones relevantes en el caso que nos ocupa.

Basaremos la respuesta en Schenker-Wicki et al. (2010), quienes distinguen dos factores productivos básicos en la gestión de una catástrofe. Estos son, la "prevención" y la "intervención" respectivamente. Tal como en una clásica función de producción, ambos factores son sustitutos uno del otro, y su exacta combinación responde a la tecnología vigente (o conocida) en torno al tema, a la experiencia acumulada sobre la incidencia (y ocurrencia) de fenómenos semejantes y a la institucionalidad que condiciona la gestión pública (y privada) en estas materias. La ilustración 1 muestra en forma gráfica los elementos del problema. Cabe entender que tanto la prevención como la intervención pueden a su vez separarse en componentes específicos, cada uno de los cuales supone una definición en cuanto al nivel de gobierno responsable y al grado de autonomía con el cual este puede actuar. Debe entenderse que tanto la prevención como la intervención serán desplegadas tanto por el Estado como así también a través de la comunidad organizada, ya sea colectivamente o a través del mercado. Una forma concreta de prevención espontánea es la transferencia del riesgo a un tercero y/o la decisión autónoma de construir viviendas antisísmicas localizadas en zonas de menor peligro. Diversos problemas de agencia, asimetrías de información y mercados incompletos podrían sin embargo inducir severas fallas en el logro de una solución socialmente óptima sin la asistencia directa del Estado (Barr, 1992). Cabe por lo tanto tener un "seguro social" que corrija estas falencias y proteja a la población de las pérdidas potenciales de una tragedia.

En medio de las dos variables básicas mencionadas, se encuentra lo que reconoceremos como el "componente aleatorio". Este posee una importancia vital, toda 
vez que la gama de incidentes graves que reconoceremos como catástrofes, puede adquirir características muy difíciles de anticipar. Los "engranajes" que generan el resultado deben interpretarse como la "tecnología" utilizada, la cual será función de variados factores. Uno de ellos es la propia organización del Estado en la materia, la cual admite múltiples formas. Otro elemento fundamental es la acción de la propia comunidad a través de acciones autónomas, ya sea en colaboración con el Estado, o en forma independiente. En ello encontraremos elementos culturales que pueden favorecer el resultado, entre los cuales se cuenta el propio "capital social' y la actitud individual y colectiva frente a la adversidad. Cabe suponer que la propia experiencia de catástrofes previas genera cierto aprendizaje, el cual es pertinente tanto a nivel social, como también en el diseño de las políticas públicas.

\section{Ilustración 1}

Variables fundamentales en la gestión de catástrofes

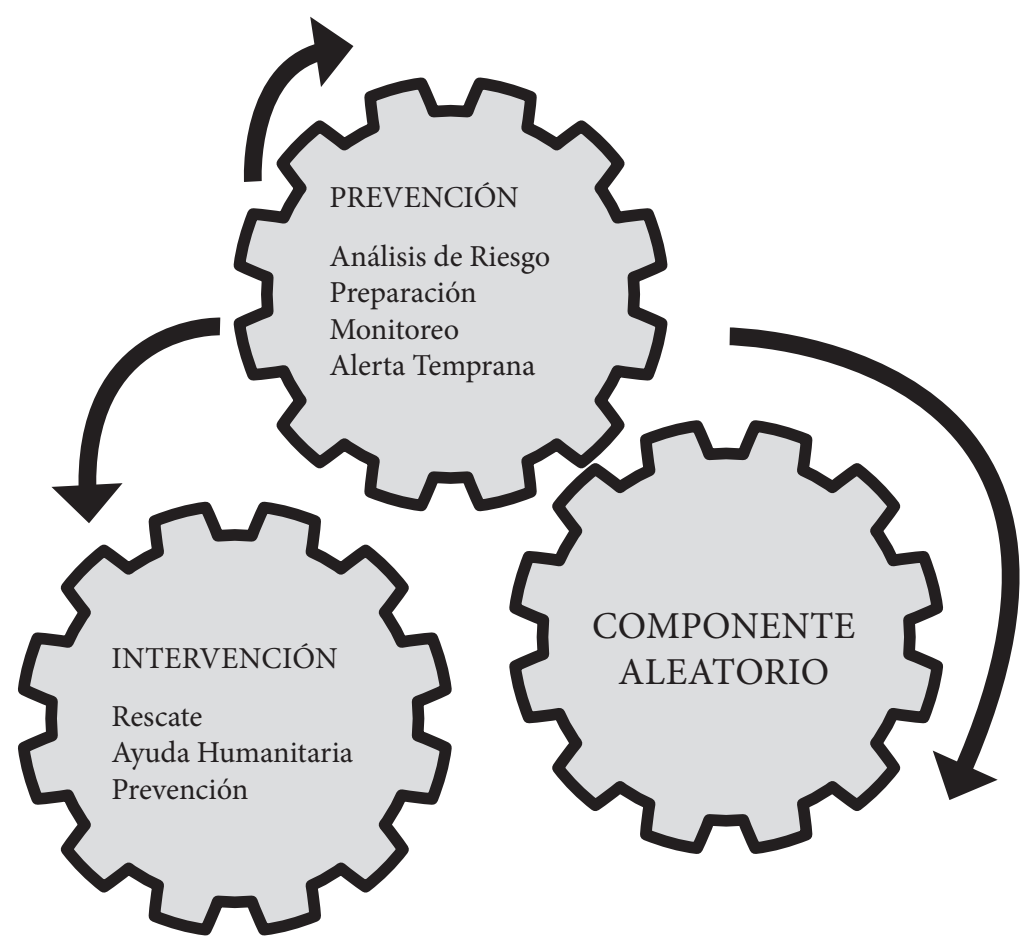


Este esquema analítico puede sintetizarse en una función de producción. Esto implica aceptar que habrá un nivel socialmente óptimo de prevención e intervención, el cual a su vez tiene una expresión concreta en cada una de las dimensiones específicas o componentes indicados en la ilustración 1. Dicha intervención (prevención) óptima deberá ser políticamente decidida, habida cuenta de los costos y potenciales beneficios de la misma. Debe ser evidente por ejemplo, que no todos los problemas de abastecimiento, información y comunicación pueden estar cubiertos, de no mediar costos imposibles de asumir. Habrá por lo tanto escenarios probables que deben evaluarse en su mérito, cada uno de los cuales supone compromisos diversos por parte de la autoridad.

La ecuación 1 (Ec. 1) resume lo antes mencionado. Mientras $P$ e $I$ representan la dosis de prevención e intervención tanto pública como privada, $Y$ es nuestra variable endógena, y ella está compuesta de dos elementos. Uno es la pérdida de vidas humanas $(\mathrm{H})$, y el segundo es el daño material causado por la catástrofe (M). En términos formales, esto significa que $Y$ será una función inversa de ambas pérdidas. El término $A$ representa la tecnología necesaria para generar $Y$. El reconocimiento de que el impacto de una catástrofe contiene inevitablemente un componente aleatorio se encuentra explícito en $u$.

$$
Y=A \times F(I, P)+u
$$

El resto del trabajo se centra en el término $A$ y más específicamente en el componente público de dicho coeficiente. En él se encuentra implícita la problemática en torno a la apropiada organización del Estado. Más concretamente, debemos preguntarnos qué unidad debe encargarse de cada concepto específico al interior de $I$ y $P$, y qué nivel de gobierno debe asumir responsabilidades en cada caso. De igual manera, los incentivos que el marco institucional vigente producen en los numerosos actores involucrados, son parte del debate en torno al marco institucional apropiado. 


\subsection{El problema de la asignación de funciones entre niveles de gobierno}

\section{El caso general}

Pocos son los aportes teóricos que iluminan el problema de la óptima distribución de responsabilidades al interior del Estado en lo que a gestión de catástrofes se refiere. La economía del sector público ha generado dos líneas analíticas alternativas que permiten abordar la pregunta genérica en torno a cómo "deben" asignarse competencias entre niveles de gobierno. Primeramente, el enfoque normativo, usualmente asociado a la figura de Richard Musgrave, aporta un conjunto de recomendaciones (normas), conducentes a una solución eficiente en lo concerniente a una intervención eficiente del Estado. Este enfoque supone que el Estado actúa como un "dictador benevolente", el cual a su vez representa e interpreta fielmente las preferencias de la comunidad. Musgrave (1958) sostiene que mientras las funciones de "estabilidad macroeconómica" y de "redistribución del ingreso" deben ser administradas por el nivel central, la llamada función de "asignación" puede ser ejecutada eficientemente por los niveles descentralizados de gobierno. Esta clasificación debe entenderse en razón de las externalidades entre jurisdicciones emanadas de las decisiones fiscales de alcance agregado, tales como el equilibrio presupuestario y/o la estabilidad de precios, y las dificultades de alcanzar el "equilibrio" en el marco de la función de equidad. Si los beneficiarios locales migran o "votan con el pie" (Tiebout, 1956) en favor de aquellas jurisdicciones más ricas, y a su vez los residentes más ricos escapan de aquellas comunidades más orientadas a la redistribución, el resultado (equilibrio) final puede ser inestable, incierto, e incluso incumplir la propia condición de que tal equilibrio en verdad "exista” en la optimización dinámica del problema.

Otros aportes en la misma línea se han centrado en el alcance geográfico de las competencias. Un concepto de interés es la idea de "correspondencia fiscal" desarrollado por Olson (1969), en virtud del cual se propone que la eficiencia asignativa se alcanza mejor en la medida que exista coincidencia entre quienes pagan por el servicio entregado y los correspondientes beneficiarios del mismo. Un experimento extremo en esta dirección es el conocido "Poll Tax", implementado en Inglaterra entre 1979 y 1982. Al igual que en la propuesta general de Musgrave, lo que está en juego en la hipótesis de Olson, es que el grado de rendición de 
cuentas, y por lo tanto de eficiencia en la provisión del servicio, solo se logran en la medida que no existan externalidades entre jurisdicciones. Si las hubiera, ello puede gatillar incentivos perversos que desvían el óptimo privado, definido e implementado por cada jurisdicción en forma descentralizada, del interés de la sociedad como un todo. Una propuesta original es aquella desarrollada por Fray (2001). Dicho autor sostiene que la provisión de bienes públicos locales debiese estar radicada en jurisdicciones unifuncionales (“distritos"), las cuales podrían competir entre sí por capturar potenciales participantes. Estas debiesen poder cobrar sus propios impuestos, y ser autónomas políticamente. Los eventuales problemas de coordinación entre jurisdicciones serían conducentes a una solución endógena del problema, por la vía de una expansión en el tamaño de tales jurisdicciones, hasta que los efectos externos de cada gobierno local así definido fuesen completamente internalizados. El aspecto más distintivo en la propuesta de Frey, es que las unidades de gobierno referidas no tendrían -necesariamenteuna soberanía territorial específica, pudiendo coexistir más de una jurisdicción dentro del mismo territorio. Casos concretos que ilustran la factibilidad de esta propuesta serían la propia gestión corporativa de las compañías trasnacionales, la cual es asimilable a un "gobierno sin territorio", y la función rectora ejercida por la religión en el marco de la organización de la iglesia.

La visión alternativa a Musgrave está encarnada en la nueva escuela institucionalista y la tradición disciplinaria del enfoque de la Elección Pública. Bajo este prisma el Estado es un Leviatán, el cual produce rentas ricardianas a favor de quienes lo administran, hecho que amerita la implementación de restricciones institucionales para contenerlo. Una de ellas es la descentralización (Brennan y Buchannan 1980), la cual diluye el poder del Estado en muchos pequeños (y más débiles) leviatanes. De lo anterior se sigue que siempre será mejor descentralizar, puesto que así reducimos la probabilidad de explotación del gobierno en ejercicio respecto de los contribuyentes. Aunque la hipótesis así definida tiene particular relevancia en el ámbito tributario, debe entenderse que cualquier forma de delegación de funciones a niveles descentralizados de gobierno produce competencia horizontal en la gestión del Estado, reduce la probabilidad rentas a favor de quienes administran el Estado y mejora su eficiencia.

Puede decirse que un rasgo común de los argumentos basados en la definición "eficiente" de las jurisdicciones subnacionales, es que debe haber la máxima 
posible coincidencia entre los beneficiarios y los que pagan por el servicio. No obstante lo anterior, la propia literatura económica reconoce que la problemática en torno a la asignación de funciones entre niveles de gobierno, no se agota en la definición de aquello que podríamos llamar la asignación óptima. Definir una solución implica abordar un aspecto crítico en la organización del Estado, uno en el cual hay distribución de poder y por lo tanto implicancias políticas evidentes (Dafflon, 2006). El debate en torno a cómo se distribuye este poder contiene ingredientes históricos, culturales y por cierto también económicos. Este ensayo intenta aislar este último componente, bajo el supuesto de que constituye un aspecto muy significativo en la conformación del propio Estado nación, y en la estructura específica de dicho Estado a la luz del problema que nos ocupa. Aunque no existe evidencia formal al respecto, sí es claro que ciertas funciones del Estado suelen estar radicadas en un determinado nivel de gobierno (Hooghe y Marks, 2009), circunstancia que arroja evidencia de primera instancia a favor de que la eficiencia ocupa al menos un lugar en el amplio rango de factores que explican la distribución efectiva del poder al interior del Estado.

\section{El caso de la función de seguridad y la prevención de catástrofes}

La función de seguridad y la prevención de catástrofes es evidentemente una responsabilidad del Estado. Por una parte, la seguridad y el manejo de crisis colectivas en las cuales está en juego la vida de muchas personas en una comunidad, tiene las clásicas características de un bien público. Por la otra, la inevitable tendencia de los individuos a postergar decisiones cuyos beneficios se manifestarán muchos años después, puede interpretarse como el fruto de una divergencia entre la tasa de descuento privada y la tasa de descuento "social". En el marco de este ensayo, procede pues preguntarse qué grado de descentralización debe haber en esta función. Bajo este prisma, la acción del Estado puede entenderse como la respuesta natural a una o más fallas del mercado, el cual -teóricamente- podría resolver este problema a través de un seguro privado.

El alcance de cualquier hipótesis sobre el particular, y sus implicancias sobre la articulación de la gestión pública ante crisis y/o catástrofes, descansa sin embargo en lo que entenderemos como descentralización. Distinguiremos tres niveles. El primero es el nivel centralizado. Entenderemos por tal un esquema en el cual los 
recursos, las normas que regulan la función en cuestión, y la localización física de la unidad administrativa correspondiente, se encuentran en la ciudad capital, o en el centro político o económico de la nación. En este esquema, los gobiernos subnacionales son meros agentes del gobierno central, y dependen de este para todos los efectos de implementación y financiamiento de sus políticas. Una segunda categoría es la llamada "desconcentración", o "federalismo disperso" en el caso de los países federales, el cual consiste en la desconcentración física de las unidades de gestión que atienden y previenen la catástrofe. Este esquema repara al menos en parte, la falta de información y potenciales problemas de coordinación que el nivel central pueda enfrentar como resultado de una catástrofe. Finalmente, un modelo descentralizado supone autonomía en la administración de los recursos y en su capacidad de decidir sobre cuestiones vitales que atañen al uso de los mismos. Su beneficio central es la mayor rendición de cuentas ante la comunidad por parte de las autoridades locales, y la mejor capacidad de reacción ante eventos que exigen información precisa y oportuna para ser debidamente atendidos.

Como hipótesis central plantearemos que las políticas públicas destinadas a prevenir e intervenir en una catástrofe enfrentan un conflicto entre dos importantes objetivos (Letelier y Sáez, 2006). Por una parte, las múltiples interrelaciones entre jurisdicciones y la inevitable necesidad de una acción conjunta a nivel nacional tanto en la etapa de la prevención como en la de intervención, implican que la centralización nos concede el beneficio de la coordinación o efecto escala. Sin embargo, mientras más coordinemos, más nos alejaremos del objetivo opuesto, el cual se constituye en el beneficio de la información. Llamaremos a este último efecto Von Hayeck. La intervención en el marco de una catástrofe exige usualmente mucha información, hecho que exige recurrir al nivel más descentralizado posible -y por lo tanto más informado- para articular la intervención. Inevitablemente, y no obstante la muy amplia gama de tipos diferentes de catástrofes, enfrentaremos un conflicto entre ambos objetivos. Este será especialmente visible en la etapa de intervención, en la cual la dimensión territorial del episodio adquiere especial importancia.

Por una parte, cada tipo diferente de crisis o catástrofe amerita una combinación diferente de coordinación e información. Los eventos referidos pueden variar según sean desastres naturales versus creados por el hombre, si son conflictos sociales o guerras civiles, o en función de la amplitud geográfica del evento o el origen 
exógeno o endógeno del mismo. Es importante también reconocer que diferentes grupos de la sociedad pueden interpretar la crisis en forma diferente. De hecho, cualquiera sea la naturaleza del problema, la connotación "crítica" de la situación es consustancial a la percepción colectiva sobre el particular, pudiendo diferir entre áreas geográficas, comunidades específicas, partidos y niveles de gobierno. Lo que para unos es simplemente un desastre económico y social, para otros puede significar una oportunidad (Rosenthal y Kouzmin, 1997). Incluso en el evento de que todos los grupos involucrados (partidos, intereses corporativos, comunidades organizadas, etc.) concuerden en que el problema es en verdad muy grave, la solución del mismo y la asignación de prioridades suele exigir la confrontación de consideraciones valoricas difíciles de compatibilizar.

\section{AlgunOS CASOS}

En el caso de Estados Unidos, dos hitos recientes cabe mencionar, los cuales han alimentado el debate sobre la apropiada preparación e intervención ante una catástrofe. El primero fue el llamado "Septiembre 11 del 2001". Antes de tal episodio, la Federal Aviation Agency (FAA) y el North American Aerospace Defense Command (NRAD) habían acordado un sistema de colaboración en caso de secuestro. Antes de que la Fuerza Aérea pudiera derribar un avión civil, el acuerdo establecía seguir un protocolo de múltiples notificaciones y aprobaciones al más alto nivel de gobierno. Por cierto, en el episodio de Sept. 11 no hubo tiempo de seguir dicho protocolo. El vuelo 11 (que impactó la torre Norte del World Trade Center), estaba siendo rastreado por la oficina de Boston de la FAA, la cual se dio cuenta del problema. En lugar de seguir el protocolo acordado con la NRAD, se comunicó directamente a la Fuerza Aérea, solicitando los F16 (Boin et al., 2005). Minutos antes de que el Vuelo 11 chocara con las torres, el vuelo 175 se perdió del radar para dirigirse a las torres. Este cambio no fue percibido, pues el controlador aéreo responsable por el vuelo 175 estaba preocupado del vuelo American 11 y no respondieron el llamado. Cuando el controlador se dio cuenta que tenían un segundo problema, un colega trató de notificar a los responsables regionales. Sin embargo, estos no estuvieron dispuestos a escuchar, pues estaban preocupados del primer choque. Entre tanto, el American 77 había desaparecido del radar 
del centro de Indianápolis. Este centro no estaba consciente del primer choque, y comenzó a buscar claves para decidir.

El segundo es el caso del Huracán Katrina en el 2005. Hasta la primera mitad de los años 50, tanto los estados como los gobiernos locales tenían un importante papel que jugar en la defensa civil, la preparación y la atención de las víctimas de un desastre. La estrategia organizacional y el foco de atención estaban muy orientados a los eventuales ataques nucleares resultantes de una guerra atómica. Solo después del Huracán Diana en 1955, el nivel federal a través de la Federal Civil Defense Admintration, se involucró más directamente en la coordinación de la ayuda a los niveles subnacionales, lo cual vino acompañado de normas comunes y por lo tanto más centralización (Robert, 2008). El reporte posterior al Huracán Katrina (Post Hurrican Katrina Report, 2005), seńala que los encargados del DHS no supieron entender las exigencias inmediatas de la tragedia. Los conflictos posteriores entre esta unidad y la propia Casa Blanca, frustraron una acción rápida y coordinada en beneficio de las víctimas. En suma, las autoridades nacionales (nivel federal), tuvieron más dificultades en la implementación y la ayuda inmediata que los estados y niveles subnacionales de gobierno.

Entre los países en vías de desarrollo la experiencia es muy diversa (Bollin et al., 2003). Entre los países unitarios de América Latina un caso muy descentralizado es el de Colombia, en el cual la Constitución de 1991 avanzó notablemente en conceder mayores grados de autonomía al nivel departamental y municipal. Un hito de importancia en lo que a gestión de catástrofes se refiere es el desastre generado por el Volcán Nevado del Ruiz en 1987, el cual gatilló la implementación de una Estrategia Nacional de Emergencias bajo la forma de un Sistema Nacional de Prevención y Atención de Desastres. Dicha iniciativa convive con una importante delegación de responsabilidades de prevención en el municipio. Como parte del sistema a nivel nacional, se crearon Comités multiinstitucionales y multisectoriales a nivel departamental y local. Sin embargo, la gran diversidad de tipos distintos de municipios y el muy heterogéneo acceso a recursos y capacidad de gestión entre ellos, ha generado gran diversidad en el esfuerzo local sobre la materia. En un extremo opuesto se encuentra Guatemala, país en el cual existe una institucionalidad al respecto desde 1996. Esta creó la Coordinadora Nacional para la Prevención de Desastres (CONRED), instancia de coordinación para la prevención y atención de las mismas. Dicha unidad tiene una versión específica 
tanto a nivel nacional como sub nacional. También en este caso, el alcance real de la norma choca con la diversidad y carencia de recursos de los municipios.

Mientras que en América Latina la normativa está muy orientada a la coordinación, una normativa más específica se puede encontrar en Filipinas. Desde 1978, este país impone por Ley a los municipios la exigencia de que cada localidad debe destinar anualmente el 5\% de su presupuesto para el llamado "Fondo Local de Calamidades", el cual es complementario al "Fondo Nacional de Calamidades".

\section{LAS DIMENSIONES DE UNA CRISIS. EL CASO DE UN TERREMOTO}

\section{1 Tres componentes básicos de la gestión}

El conjunto de conceptos, hipótesis y normas basadas en la teoría, debiesen tener la suficiente amplitud como para cobijar formas de acción del Estado tan diversas como son la administración de la educación, la gestión de la salud pública, la provisión de servicios viales, sanidad y también -por diferente que sea- el manejo de una crisis. Al respecto cabe reiterar la naturaleza multidimensional de cada función del Estado. Este rasgo general también incluye al tema que nos ocupa. Cualquiera sea su origen, el manejo de una crisis supone atender problemas políticos, administración de recursos físicos y humanos, mantención del orden y otros varios. No basta por lo tanto señalar que dicha gestión debe estar radicada en un determinado nivel de gobierno. Debemos también precisar la dimensión específica de la cual hablamos, y evaluar los méritos del caso en el contexto de factores institucionales, culturales y económicos.

En el caso de un terremoto, o un desastre natural severo, hay por lo menos tres rasgos específicos que adquieren especial relevancia. Uno primero, está asociado con la necesidad de entregar ayuda humanitaria a los necesitados (ilustración 1), lo cual puede involucrar un importante problema logístico. La destrucción de infraestructura básica y la falta de comunicaciones entre unidades de gobierno es un problema central en un terremoto de gran magnitud. El segundo elemento, más vinculado al concepto de "rescate", es la restitución del Estado de derecho si este llegara a perderse. Este probó ser un problema muy relevante en el caso del 
terremoto del 27 de febrero en Chile en la ciudad de Concepción, como lo ha sido también en la experiencia de Haití. Finalmente, la oportuna señal de una alerta temprana representa un factor clave en el desenlace. Miraremos estos tres aspectos en la experiencia chilena.

\subsection{El caso del Terremoto del 27 de febrero en Chile}

\section{Prevención}

Habremos de preguntarnos las dos cuestiones básicas que dan forma a una política pública. La primera es cuántos recursos destinaremos al fortalecimiento de las dos variables explicativas de la Ec.1. Inevitablemente, la respuesta tiene un componente político importante, pero está mediatizada por consideraciones técnicas e históricas del país y región específica de que hablamos. Si bien son varios los organismos y unidades administrativas del sector público chileno involucradas en la prevención e intervención en el caso de una catástrofe, es la llamada "Oficina Nacional de Emergencia” (ONEMI), el organismo cuya misión está expresamente orientada a la función de prevención e intervención antes referida. De acuerdo con la Ley de presupuestos del año 2010, la ONEMI recibirá este año recursos cercanos a los US\$14.000.000, y su misión consiste en "Planificar, impulsar, articular y ejecutar acciones de prevención, respuesta y rehabilitación frente a situaciones de riesgo colectivo, emergencias, desastres y catástrofes de origen natural o provocados por la acción humana, a través de la coordinación del Sistema Nacional de Protección Civil para la protección de las personas, los bienes y el ambiente". Este mandato amplio, deja por definir qué acciones concretas debe abordar la ONEMI, y qué grado de tuición tiene sobre las unidades del Estado que teóricamente debiesen participar en el cumplimiento de este objetivo.

La segunda pregunta es cómo lo gastamos. Más concretamente, cómo se organiza el Estado para abordar esta importante función. La ONEMI depende del Ministerio del Interior y su estructura administrativa puede considerarse "desconcentrada", toda vez que al menos desde el 2006, este organismo posee secretarías regionales en cada región. En esta perspectiva, la acción de la ONEMI puede solo llegar hasta el nivel provincial (gobernación), quedando fuera de su acción directa la esfera propiamente municipal. Ello supone una dificultad práctica importante, toda vez que una 
catástrofe (e.i. un terremoto), posee una dimensión territorial que se expresa en el ámbito municipal. De acuerdo a la Ley Orgánica Constitucional de Municipalidades, estas últimas poseen dos funciones que guardan estrecha vinculación con sendos componentes de la Ec.1 (I,P). Primero, deben "Aplicar las disposiciones sobre construcción y urbanización, en la forma que determinen las leyes, sujetándose a las normas técnicas de carácter general que dicte el ministerio respectivo”. Dado que tales normas están centralmente definidas, puede considerarse que en esta materia el municipio actúa como un agente del gobierno central. Sin embargo, de acuerdo con el Art. 4 de la misma Ley, el municipio debe también colaborar en "la prevención de riesgos y prestación de auxilio en situaciones de emergencia o catástrofes". De lo anterior se sigue que el nivel local de gobierno posee una potencial injerencia en labores de rescate a las víctimas de un terremoto, asistencia a los damnificados y prevención de situaciones de riesgo.

Respecto de los organismos sobre los cuales el nivel central (ONEMI) sí tiene una injerencia directa, la función del gobernador (provincial) es amplia y se inserta en una función desconcentrada del nivel regional, del cual depende. Entre sus atribuciones, se encuentra bastante perfilada aquella relativa al "orden público y la seguridad de sus habitantes y bienes", y la de "requerir el auxilio de la fuerza pública en el territorio de su jurisdicción, conforme a la Ley”. Debe entenderse por lo tanto, que en la etapa de “intervención" resultante del terremoto del 27 de febrero, el gobernador tuvo un importante papel que jugar. Cabe mencionar que estas funciones son sin embargo compartidas con el nivel regional, el cual entre otras materias, tiene competencias para convocar a la fuerza pública en caso de que esto sea necesario.

Atendiendo a la evidente amplitud de las normas vigentes, procede examinar cómo se articula la gestión de la ONEMI en el marco de su misión institucional. $\mathrm{Al}$ respecto cabe mencionar que una instancia muy importante en la etapa de preparación ante un evento catastrófico, es la conformación de los llamados Comités de Protección Civil (CPC). Estos son patrocinados por la ONEMI en el marco del DL. 156 del 2002 y tienen una versión a nivel de gobierno central, y capítulos regionales, provinciales y municipales. En ellos se encuentran representados quienes son los protagonistas naturales de una emergencia. A saber, las fuerzas policiales, el voluntariado (bomberos), los encargados del rescate en base a ambulancias, las fuerzas armadas y otros miembros relevantes de la sociedad civil. Solo cuando 
se produce una catástrofe, dichos comités se transforman en operativos (Comités de Operaciones de Emergencia: COE), y se constituyen en el canal que transmite información a la ONEMI en torno a las necesidades reales de la población local. Esta fluye primero desde la comuna a la gobernación, y desde allí se consolida a través de la intendencia para llegar luego al nivel central. Un rasgo clave del terremoto del 27 de febrero, es que tal información fue difícil de recopilar, puesto que la propia constitución de los COE fue en algunos casos físicamente imposible, y en otros la propia información de base no pudo ser transferida oportunamente al nivel superior.

Entendida la acción de la ONEMI como una instancia de coordinación entre unidades de gobierno, una cuestión central es cómo logra este organismo que el nivel municipal, el cual goza de autonomía política y administrativa, colabore en forma efectiva en la generación de la información requerida. La respuesta está en el hecho de que si el nivel local (municipio) no presta la debida atención al COE, y no se ciñe a los protocolos establecidos en el Plan Nacional de Protección Civil, la ONEMI no hace llegar la ayuda correspondiente a este municipio. Lo anterior se ve agravado por la inexistencia a nivel municipal de un Director de Protección Civil, hecho que introduce un vacío importante en la relación de la ONEMI con el nivel local. Una cuestión a definir es la eventual dependencia jerárquica de este funcionario, toda vez que la dependencia del nivel central (gobernación, intendencia, ONEMI), puede tener grandes implicancias en la capacidad de articular acciones destinadas a socorrer a las víctimas de una tragedia.

La asistencia de las Fuerzas Armadas se expresa en tres ingredientes básicos. El primero, de gran importancia en el evento del 27 de febrero, es la generación de información. En el caso de un maremoto, esta función recae en el "Servicio Hidrográfico y Oceanográfico de la Armada” (SHOA). Este posee múltiples funciones orientadas a la generación de la información necesaria para facilitar la navegación en forma segura. Un aspecto crítico de su misión es el de "proporcionar los elementos técnicos y las informaciones y asistencia técnica destinada a dar seguridad a la navegación en las vías fluviales y lacustres, aguas interiores, mar territorial y en la alta mar contigua al litoral de Chile".

Lo anterior implica que este organismo posee una responsabilidad ineludible en facilitar la información que la ONAMI requiere para proceder a intervenir en 
labores de rescate y asistencia en caso de una catástrofe marítima. El segundo aspecto se refiere a la mantención del orden público. De acuerdo a la Ley orgánica Constitucional de Estados de excepción, la eventualidad de una crisis con características de catástrofe, habilita al Presidente (a) de la República a declarar la zona afectada en "estado de catástrofe", lo cual supone delegar en el jefe militar de la zona, las funciones y competencias regularmente ejercidas por la autoridad política. Finalmente, las fuerzas armadas deben también participar en la búsqueda y salvamento de las víctimas de una catástrofe (D.S. 375). En la actualidad, las fallas en el sistema de alerta a cargo del SHOA están siendo revisadas en el marco de un sumario. Sin embargo, es importante señalar que la información utilizada por el SHOA en el caso de un terremoto y/o maremoto, es facilitada por el Centro de Alertas del Pacífico con sede en Hawai.

\section{Intervención}

Dos elementos centrales probaron ser críticos en la etapa de intervención. El primero se refiere a la organización básica del abastecimiento de insumos y productos esenciales para atender una emergencia. Esta está articulada en base a la acción de la ONEMI, en colaboración con las fuerzas armadas y la propia sociedad civil. En lo que a la acción de la ONEMI se refiere, este organismo mantiene una bodega central en Santiago, y cuatro bodegas regionales en Iquique, Copiapó, Concepción y Puerto Aysén. Cada una mantiene frazadas, planchas de zinc, generadores, cocinillas y una dosis de alimento básico para una familia de cuatro personas por cuatro días. Hay una dirección regional en cada región, equipos de radio y sistemas de monitoreo. Tal como fue mencionado anteriormente, la solicitud formal de la ayuda respectiva parte del nivel comunal (local), instancia que informa a la gobernación (provincia), la cual reporta el requerimiento correspondiente a la intendencia (región). El procedimiento concluye con la evacuación de una solicitud formal de un "Fondo de Emergencia" (FEMER), el cual especifica los requerimientos materiales y financieros del caso. Respecto a la distribución de insumos no disponibles en las bodegas, estos deben comprarse. Este fue un aspecto crítico del terremoto del 27 de febrero, puesto que dichas compras no pueden ser licitadas por motivos obvios. Para estos efectos, la Ley de Compras del Estado establece que se puede recurrir a la adquisición por la vía de un "convenio marco", siempre que este incluya aquellos elementos demandados por la emergencia, 
y/o la modalidad de trato directo, la cual exime del procedimiento de licitación (Ley 19.886). Una vez más, el daño de la infraestructura básica, y de las propias unidades productivas encargadas de producir y vender tales insumos, impidieron la debida provisión de tales insumos y su correspondiente distribución entre los afectados. Ello agregó una dificultad adicional en le etapa de intervención.

El segundo aspecto tiene que ver con la seguridad pública. En condiciones de catástrofe, las fuerzas policiales en las zonas afectadas no pudieron evitar las acciones de saqueo y vandalismo posteriores al terremoto. Una posible reflexión es que nuevamente en este caso, se produjo una notable asimetría de información entre las autoridades políticas centrales, y la propia realidad de las ciudades en las cuales tuvo lugar dicho saqueo. Ello apunta claramente en la dirección de que alguna forma de descentralización es requerida en el proceso conducente a decidir la declaración de estado de catástrofe si es que las circunstancias lo ameritan. En esta misma perspectiva, parce lógico pensar que las medidas de excepción contempladas en la Ley, y que utilizan fuerzas militares a fin de colaborar con las fuerzas de orden regulares, debiesen admitir un rango más amplio de opciones, entre las cuales alguna puede ser de resorte regional.

\section{CONCLUSIONES}

La acción del Estado en la prevención e intervención de catástrofes se justifica en razón del carácter meritorio de tal intervención y de las fallas inherentes al mercado de los seguros privados, los cuales no resguardan a la población civil ante situaciones extremas provocadas por fenómenos naturales. Problemas de información, riesgo moral y selección adversa son algunos de los factores más evidentes.

La función de producción que genera seguridad ante eventos catastróficos posee dos factores productivos. Estos son la "prevención" y la posterior "intervención". La tecnología utilizada para combinar los esfuerzos en estos dos ámbitos contiene elementos privados y públicos, toda vez que las acciones inherentes a los dos factores mencionados son desplegadas simultáneamente por el Estado y la Sociedad Civil organizada. Existe sin embargo un componente aleatorio, el cual puede ser muy importante e inesperado en el resultado final. 
En el caso del terremoto del 27 de febrero en Chile, los actores claves fueron la ONEMI, el SHOA y la propia acción del ejecutivo a través de las decisiones tomadas en el nivel central. Aspectos críticos en los resultados fueron la falta de información, la descoordinación entre la acción del SHOA y de la ONEMI y las dificultades de comunicación con las regiones afectadas inherentes a la tragedia. Sin perjuicio de las evidentes debilidades en los flujos de información necesarios para atender e identificar a los afectados, los problemas de saqueo y desorden públicos en Concepción, deben hacer reflexionar sobre la factibilidad de descentralizar ciertas decisiones en torno a la intervención. Más concretamente, la facultad de declarar estado de catástrofe, y de utilizar las Fuerzas Armadas como un refuerzo de la fuerza pública regular, deben ser un resorte administrativo del nivel intermedio de gobierno, el cual -en el caso de Chile- es una unidad administrativa desconcentrada del gobierno central. Dada la fuerte connotación política que posee la condición de Estado de Catástrofe en la legislación chilena, una solución factible es la posibilidad de solicitar la colaboración de las Fuerzas Armadas en el marco de un estado de emergencia menos severo y radical que el "estado de catástrofe".

\section{REFERENCIAS BIBLIOGRÁFICAS}

Barr, N. (1992). "Economic Theory and the Welfare State: A Survey and Interpretation”, Journal of Economic Literature, vol. 30, pp. 741-803.

Boin A., 'T Hart P., Stern E. y Sundelius B. (2005). The Politics of Crisis Management. Public Leadership under pressure. New York: Cambridge University Press.

Bollin C., Cárdenas C., Hahn H. y Vatsa K. (2003). Gestión de Riesgo de Desastres por Comunidades y Gobiernos Locales. Serie de Estudios Diálogo Regional de Política. Banco Interamericano de Desarrollo, Washington D. C.

Brennan G. y Buchanan J. (1980). The Power to Tax. Analytic Foundations of a Fiscal Constitution. New York, Cambridge University Press.

Dafflon B. (2006). "The assignment of functions to decentralized government: from theory to practice", en Ahmad E. y Brosio G. (Eds.) Handbook of Fiscal Federalism, Edward Elgar. 
Fray B. (2001). "A Utopia? Government without Territorial Monopoly". The Independent Review, Vol. VI, N 1, pp. 99-112.

Hooghe L. y Marks G. (2009). “Does Efficiency Shape the Territorial Structure of Government?”. Annual Review of Political Science, pp. 225-241.

Letelier S. L. y Sáez L. J. L. (2006). “Economies of Scale, Information and Fiscal Decentralization”, Proceeding of the IIPF Conference on Public Finance. Paphos: Chipre.

Ministerio del Interior. (2002). Plan Nacional de Protección Civil. Decreto Ley 156. Santiago: Gobierno de Chile.

Musgrave R. (1958). The theory of public finance. London: McGraw-Hill.

Olson, M. (1969). The principle of Fiscal Equivalence: The division of Responsabilities among Different Levels of Government, American Economic Review Papers and Proceedings, No 59, pp. $479-487$.

Robert P. (2008). "Dispersed Federalism as a New Regional Governance for Homeland Security", Publius: The Journal of Federalism, vol. 38 number 3, pp. 416 - 443.

Rothensal U. y Kouzmin A. (1997) "Crises and Crisis Management: Toward Comprehensive Government Decision Making”, Journal of Public Administration Research and Theory, pp. 277-304.

Schenker-Wicki A., Inahuen, M. y Olivares M. (2010). "Unmastered risks: From crisis to catastrophe. An economic and management insight". Journal of Business Research, pp. 337-346.

Tiebout, C.M. (1956). 'A Pure Theory of Local Expenditures', Journal of Political Economy, Vol. 64, October, pp. 416-24. 